IV Congreso Internacional Estética y Política Poéticas del desacuerdo para una democracia plural 16 y 17 de octubre. Valencia

Doi: http://dx.doi.org/10.4995/CEP4.2019.10541

\title{
Perspectiva de género y activismo político en las prácticas artísticas contemporáneas
}

\author{
Mau Monleón Pradas (Elena Edith)
}

Profesora Titular Universidad Politécnica de Valencia, Dpto. Escultura, Facultad de Bellas Artes, Laboratorio de Creaciones Intermedia, emonleon@esc.upv.es

\begin{abstract}
This analysis is based on a research project carried out in recent years around the sexual division of work and education. Globalization has empowered activist and feminist movements that cross borders, such as \#metoo, which gave voice and visibility to abuses committed against women in the workplace.

Working collaboratively and participatively, the \#EqualWorkEqualRights project is formed as an awareness campaign. For this, a methodology of knowledge is used, tracing the curriculum vitae of men and women from different continents, intertwined by the common of their writing from a gender perspective that denounces inequalities in work and education.

The practices of self-representation are present in a version of the projective curriculum vitae, which builds the future from the immediate present to reveal the aphorisms of the global capitalist economy founded on hetero-patriarchy. An activist archive is thus founded from a feminist perspective of the millennial people most affected by the precarious and gender inequalities.
\end{abstract}

Keywords: art, activism, campaign, politics, perspective, gender, work, sex, education, globalization

\begin{abstract}
Resumen
Este análisis parte de un proyecto de investigación realizado durante los últimos años en torno a la división sexual del trabajo y la educación. La globalización ha facultado movimientos activistas y feministas que traspasan fronteras, como \#metoo, que permitió dar voz y visibilidad a los abusos cometidos contra las mujeres en el mundo laboral.

Trabajando de forma colaborativa y participativa, el proyecto \#EqualWorkEqualRights se constituye en forma de campaña de sensibilización. Para ello se acude a una metodología del conocimiento situado, trazando los curriculum vitae de hombres y mujeres de distintos continentes, entrelazados por el común de su escritura desde una perspectiva de género que denuncia las desigualdades en el trabajo y en la educación.

Las prácticas de la autorepresentación están presentes en un versionado de currículum vitae proyectivo, que construye el futuro desde el presente inmediato para delatar las aporías de la economía capitalista global fundada en el heteropatriarcado. Se cimenta de esta forma un archivo activista desde una perspectiva feminista de las personas milenials que más afectadas se ven por el precariado y por las desigualdades de género.
\end{abstract}

Palabras clave: arte, activismo, campaña, política, perspectiva, género, trabajo, sexo, educación, globalización 


\section{Introducción}

Nuestra investigación se inscribe en el contexto de la estética y las prácticas artísticas, concretamente en la relación que se establece entre práctica artística y activismo político, explorando diversas metodologías, entre ellas la del conocimiento situado, trazando los currículum vitae de hombres y mujeres de distintos continentes, entrelazados por el común de su escritura desde una perspectiva de género que denuncia las desigualdades en el trabajo y en la educación.

Este texto tiene como objetivo principal realizar una exégesis y un análisis del proyecto titulado \#EqualWorkEqualRights. Sobre la división sexual del trabajo y la educación*, iniciado en 2018, y que se prolonga como Work in Progress en el espacio y en la esfera pública a través de las redes sociales. Se trata de un proyecto participativo que incluye la instalación, y que toma la forma de campaña de sensibilización dentro del arte público.

Nuestra exploración parte de un proceso de investigación llevado a cabo durante los últimos años en torno a las relaciones entre mujer, arte y trabajo en la globalización (Monleón, 2014, 2015, 2017), y se concreta en un movimiento activista y feminista que traspasa fronteras y permite dar voz y visibilidad a los abusos cometidos contra las mujeres, principalmente en el mundo laboral. Son también objetivos del proyecto visibilizar el trabajo doméstico y de cuidados realizado principalmente por mujeres, así como atender a la voz de mujeres y hombres desde la práctica del activismo político a través del arte.

En el contexto de la globalización se están produciendo enormes transformaciones en el tejido social global, así como en nuestros imaginarios colectivos. Estamos asistiendo a la crisis de la familia patriarcal; al surgimiento de nuevos modelos familiares; a la sustitución de la ética del trabajo por la ética del consumo; a la pérdida de derechos sociales; al debilitamiento de la política frente a los poderes financieros; a la precarización de la ciudadanía; y a la posición dominante de las nuevas tecnologías en nuestras sociedades. En definitiva, se trata de la crisis del modelo de sociedad del bienestar que se había empezado a gestar tras la Segunda Guerra Mundial (Cobo, 2005).

Observamos cómo la igualdad es la gran perjudicada en la base central del proyecto político de la globalización. Las mujeres accedemos al mercado laboral de forma asimétrica respecto a los varones debido principalmente al "impuesto reproductivo" (Cobo, 2005) criticado por la economía feminista (Pérez, 2014). La globalización no ha sido capaz de superar la antigua ordenación de las esferas socio-económicas definidas por la división sexual del trabajo productivo y reproductivo. Muy al contrario, si bien la mujer se ha incorporado al mercado laboral productivo de forma masiva, no ha dejado de realizar las tareas reproductivas invisibles (el trabajo de cuidados que permite la reproducción de la vida).

Nuestro proyecto \#EqualWorkEqualRights se propone visibilizar la situación laboral discriminatoria hacia las mujeres a nivel global, que comienza con la división sexual en el trabajo y en la educación, Las mujeres sufren una doble y triple discriminación (Parello, 2004 y Monleón, 2010), reflejada en gran medida en la necesidad de transitar entre el trabajo productivo y reproductivo, donde además de ser madre, se es trabajadora y, a menudo, migrante.

\subsection{Introducción a las premisas del proyecto}

El proyecto \#EqualWorkEqualRights. Sobre la división sexual del trabajo y la educación parte de la idea de que la división sexual en la educación está presente de facto en signos internos y externos en todas las culturas, como son los nombres de los colegios, los uniformes y la educación separada por sexos tanto en escuelas públicas como en privadas todavía en muchos continentes. También se parte de la propia discriminación hacia las mujeres en las universidades como, por ejemplo, en lo que se aprende y cómo se aprende: libros únicamente escritos por hombres; referentes femeninos inexistentes; techo de cristal en la dirección tanto de escuelas como de las propias universidades, y especialmente, segregación y discriminación en los estudios científicos.

La cuestión de la educación se pone en el punto de mira para el acceso a los trabajos, ya que sabemos que si bien una mayor educación aumenta las ganancias generales de las mujeres, no cierra significativamente la brecha salarial de género. En cada nivel de logro académico, los salarios medios de las mujeres son menores que los de los hombres en al menos un 21 por ciento. Además, en un análisis de 2012, (Corbett, C. y Hill, C., 2012) se descubrió que a las mujeres solo se les paga el 82 por ciento de lo que ganan sus pares varones apenas un año después de graduarse en la universidad. Diez años

2019, Universitat Politècnica de València $(c)$ BY-NC-ND 
después de la universidad, la brecha se amplía y las mujeres ganan apenas el 69 por ciento de lo que ganan los hombres. Sumado a ello, la mayoría del trabajo reproductivo no remunerado es realizado por mujeres, lo que dificulta y a menudo impide el acceso y la continuidad en otros trabajos, debido a la imposibilidad de conciliación. Se penaliza a la mujer y el trabajo reproductivo, poniendo énfasis únicamente en la productividad económica y denigrando e invisibilizando la esfera reproductiva.

Al mismo tiempo, la cultura mediática, la televisión, las películas, las series, e internet, nos imponen, bajo los mandatos de género, lo que las mujeres debemos hacer, ser y representar socialmente. Esta educación reafirma la discriminación y la violencia hacia las mujeres, como lo es la división entre sexo y trabajo en la esfera pública y la privada, así como en la dificultad de acceso y los tipos de trabajo a los que las mujeres podemos optar. En síntesis, se promueven las desigualdades en el mercado laboral y estas tienen una relación directa con la feminización de la pobreza.

Si bien el objetivo inicial del proyecto \#EqualWorkEqualRights, ha sido el de debatir la cuestión de la igualdad formal entre hombres y mujeres, a través de la investigación concreta sobre los indicadores de desigualdad laboral real en diferentes países, un objetivo más general y profundo que subyace es una crítica constructiva al sistema capitalista que promueve esta división sexual en el trabajo y en la educación.

El proyecto \#EqualWorkEqualRights se convierte así en una propuesta formal de cambio en la educación para que repercuta directamente en la esfera laboral, y de forma más general, en la lucha por la igualdad entre hombres y mujeres. Concretamente, la propuesta enfatiza la idea de que es urgente incluir la perspectiva de género en todos los ámbitos del conocimiento, desde la escuela primaria hasta las enseñanzas universitarias, para extenderla hacia todas las esferas de las relaciones interpersonales y de la comunicación.
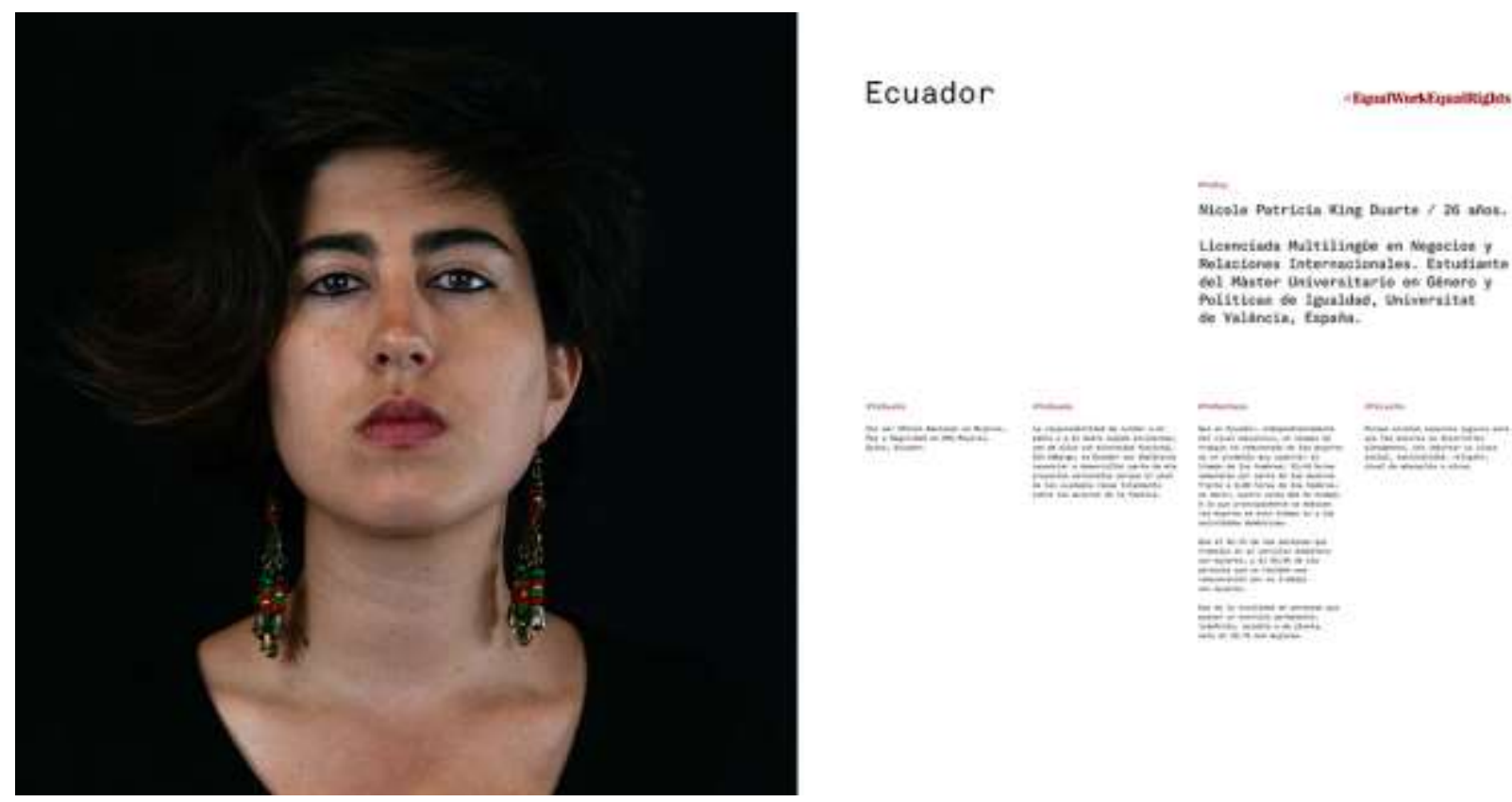

Fig. 1 Mau Monleón Pradas, \#EqualWorkEqualRights. Sobre la división sexual del trabajo y la educación,

1er ACTO. CURRICULUM VITAE, Nicole King Duarte / Ecuador, 2018.

\subsection{1er Acto / Curriculum vitae}

\#EqualWorkEqualRights es un proyecto participativo que adquiere la forma de una instalación específica para la Sala de la Muralla del Colegio Mayor Rector Peset de Valencia, (España), así como una intervención de arte público en los muros del casco histórico de la ciudad y otros espacios físicos y virtuales, como la red de internet. El proyecto tiene un carácter 
participativo y activista, y surge a partir de una comunidad temporal concreta de 130 estudiantes universitarios, de entre 18 y 45 años de edad, residentes en el mencionado Colegio, durante el curso 2017-18 (50\% mujeres y 50\% hombres) con la que se realizó un taller en el que participaron 19 personas.

Esta pequeña comunidad aporta la memoria de sus cuerpos a través del conocimiento situado, que según la antropóloga social Teresa del Valle, atesora los hitos y las encrucijadas que recorren a través de sus autobiografías. La autobiografía está presente en el proyecto a través de un 1er acto titulado currículum vitae. Este está formado por una fotografía-retrato del rostro, así como por la escritura de un currículum vitae, escrito desde una perspectiva de género.

Bajo el objetivo de presentar el futuro desde el presente inmediato para delatar las aporías de la economía capitalista global fundada en el heteropatriarcado, se cimenta un archivo activista desde una perspectiva feminista de las personas milenials que más afectadas se ven por el precariado y por las desigualdades de género. Esto es posible en el acto en que cada estudiante se entrega a imaginar y proyectar su sueño en el deseo de su futura profesión, al mismo tiempo que lo confronta con los indicadores de desigualdad en cada uno de los países soñados y en cada una de las profesiones nombradas. A favor de una concienciación y de un empoderamiento, tanto las mujeres como los hombres han escrito la historia de hoy desde la pedagogía del deseo y desde la dialéctica marxista crítica y feminista. De esta forma se produce una conciencia plena de las desigualdades entre ellos y ellas, que versan sobre las diferencias en la educación y en el trabajo. \#YoSueño, \#YoAsumo, \#YoRechazo, \#YoLucho, son los hashtags seleccionados para elaborar este currículum que busca conectar la autobiografía con la autorrepresentación en la era de las personas nativas digitales. A estos hashtags se añade la excepción del \#YoDefiendo, propuesto por la estudiante cubana Laura Bueno, en relación a la especial situación de las mujeres en su país de origen. Finalmente el \#YoLucho expresa, en una única sentencia del currículum, la voluntad de cambio, y es la llama que conecta el deseo personal con el deseo de transformación político.

Los y las participantes de los 10 países implicados: Honduras, España, Ecuador, Sáhara Occidental, Colombia, Cuba, Guatemala, Nicaragua Marruecos y Senegal, nos ofrecen una muestra cualitativa del machismo mundial, expresado en la brecha salarial, el techo de cristal, y otras desigualdades entre hombres y mujeres en el contexto laboral, mediante el estudio de las cifras concretas de cada profesión elegida. A partir de estas cifras se ha elaborado su currículum, así como un libro de fuentes que contiene sus investigaciones personales.

El trabajo reproductivo y de cuidados no remunerado está enunciado desde los principios de la economía feminista, formulando la dificultad de ser persona cuidadora de mayores, menores y personas con diversidad funcional. También se expone el estigma de ser madre y esposa, o padre y esposo, responsable de las tareas reproductivas no reconocidas ni remuneradas. De esta forma se visibiliza el trabajo doméstico y de cuidados realizado principalmente por mujeres, atendiendo a la voz de mujeres y hombres.

De forma paralela a esta lucha micropolítica ha sido importante confrontar el contexto histórico español en relación al Colegio Mayor Rector Peset de Valencia. Este está dedicado al represaliado y asesinado rector valenciano republicano Dr. Peset. Miles de mujeres fueron torturadas, violadas, maltratadas, y asesinadas durante la guerra y el franquismo pero no hemos construido una memoria histórica de mujeres emblemáticas mediante nombres de calles, colegios mayores y otros hitos y monumentos. Algunas mujeres han sido actualmente visibilizadas de su pasado en el exilio, como la periodista María Luz Morales, la actriz María Casares, la pintora Maruja Mallo, o Clara Campoamor.

Todas ellas fueron mujeres luchadoras en un contexto de extremas desigualdades en el acceso a la educación y al trabajo. Por ello, la historia de estas mujeres conecta directamente con la de Vicenta Valls Ballester, primera mujer que en el contexto republicano consiguió la Licenciatura en Medicina y Cirugía por la Universidad de Valencia y única mujer presente en la orla del curso 1930-31, año en el que fue decano de Medicina el posteriormente nombrado rector, Joan Peset.

Mi encuentro con Vicenta Valls Ballester a través de su orla de licenciatura perteneciente actualmente al Arxiu Històric de la Universitat de València y colocada en el hall del Colegio Mayor me impuso esta confrontación entre 1931 y 2018 en España. Ella es elevada a protagonista anónima de una lucha por el cambio y la igualdad en el trabajo y en la educación en la historia reciente de nuestro país. 


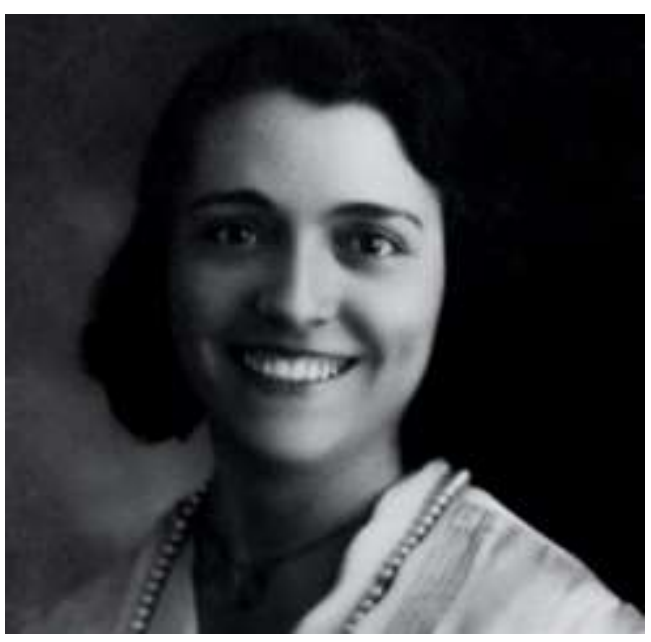

Fuente: Monleón, M (2018)

Fig. 2 Mau Monleón Pradas, \#EqualWorkEqualRights. Sobre la división sexual del trabajo y la educación ,

1er ACTO. CURRICULUM VITAE, Vicenta Valls Ballester / España, 2018. (fragmento)

\subsection{2do Acto / Campaña de sensibilización}

La Enmienda de Igualdad de Derechos, conocida por su acrónimo en inglés ERA (Equal Rights Amendment) fue escrita por Alice Paul en 1923 y propuesta a la Constitución de Estados Unidos en el Congreso, pero no llegó a ser ratificada. Tras una campaña de diez años que polarizó el debate público en muchos estados, el 30 de junio de 1982 expiró el plazo para su ratificación. Por otra parte, Equal pay for equal work [Salario igual para trabajos iguales] es un concepto conocido de los derechos laborales según el cual las personas en el mismo lugar de trabajo reciben el mismo salario. Es más comúnmente utilizado en el contexto de la discriminación sexual, en relación con la brecha salarial de género. \#EqualWorkEqualRights. Campaña de sensibilización frente a las desigualdades de género en el trabajo y en la educación retoma estos dos conceptos enfocándolos hacia el campo de los derechos humanos para centrar su atención en la igualdad real de oportunidades entre hombres y mujeres, tanto formativa como laboralmente.

El 2 do Acto del proyecto se enuncia en la forma de Campaña de Sensibilización tanto en el marco del espacio público como en las redes sociales. Se trata de la parte más activista de la producción, ya que a partir de la participación y la difusión en la construcción de imágenes "publicitarias" se establece el marco para generar cambios macro y micro políticos. La campaña parte de la deconstrucción de los currículums vitae y se centra en el ámbito tanto de los roles asignados a las mujeres como en los distintos indicadores de desigualdad. Se propone a nivel internacional, traducida en los idiomas de los países de origen de los y las participantes, llegándose a construir 5 modelos de mensaje a partir de la selección de 5 protagonistas y sus propias declaraciones, que han sido mostrados en los muros de Ciutat Vella, Barri del Carmen de Valencia (2018), así como en los mupis de la UPV en el contexto de la celebración del día Internacional de la Mujer Trabajadora (2019).

La Campaña abarca una problemática que nos afecta a todos y todas: parte de las últimas vindicaciones tanto sindicales como de las mujeres en el siglo XXI para superar las desigualdades derivadas de la división sexual del trabajo y la educación en la esfera pública y privada y se dirige tanto a varones como a mujeres, proponiendo una mirada a otras posibles economías como son las sociedades del bienestar nórdicas, o la propia filosofía de la economía feminista y la economía del bien común.

En resumen, tanto la primera parte del proyecto dedicada al currículum vitae como la segunda, a la Campaña, proponen acciones transformadoras desde dentro, ya que al realizar los talleres y las acciones participativas con los y las colegiales, se promueve el cambio desde el interior de la persona, al tener que tomar conciencia sobre la educación y el futuro laboral y utilizar la política del cuerpo y de la autobiografía. El hecho de que en la exposición se halle un espejo con el hastag \#EqualWorkEqualRights junto con una invitación a subir a las redes sociales tu propia historia te invita a intervenir sobre él y de la Campaña de Sensibilización nace la participación y su alcance entre la ciudadanía. En esta campaña puede participar cualquier persona a través del hashtag propuesto; asimismo, participan directamente asociaciones de Mujeres 
como MAV Mujeres en las Artes Visuales, Alanna Asociación Empresa de Inserción, ACVG Arte Contra la Violencia de Género, entre otras que se pueden ir sumando al proyecto en proceso. En este proyecto el arte se sitúa en la práctica del activismo político para sensibilizar e incidir en las micro y macro políticas.

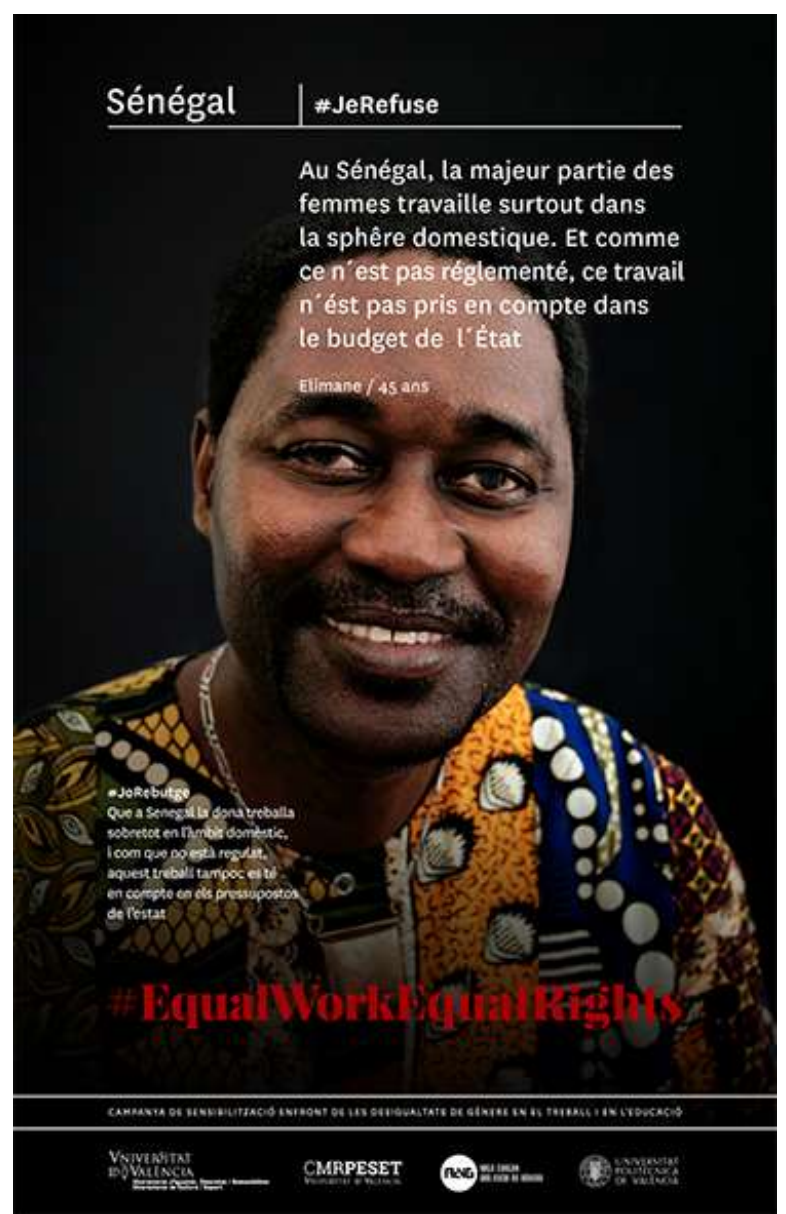

Fuente: Monleón, M (2018)

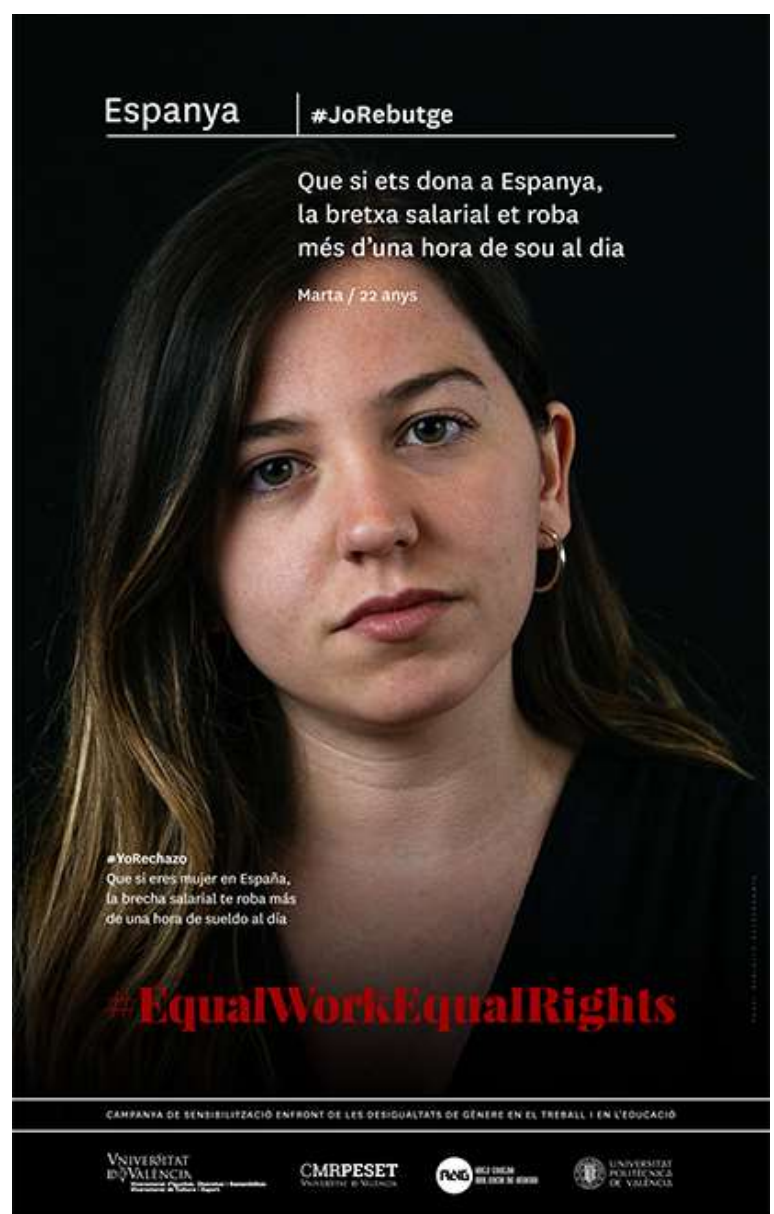

Fuente: Monleón, M (2018)

Fig. 3 y 4 Mau Monleón Pradas, \#EqualWorkEqualRights. Sobre la división sexual del trabajo y la educación,

2DO ACTO. Campaña de sensibilización frente a las desigualdades de género en el trabajo y en la educación, Elimane Nguirane / Senegal, 2018 (izquierda) y Marta Marcos Ribes, 2018 / España (derecha)

El proyecto comenzó con la palabra igualdad, en una pequeña sala-seminario del Colegio Mayor Rector Peset con un pequeño grupo de estudiantes residentes. De aquel momento cabe destacar la importante decisión que tuvimos en el taller de incluir a varones y mujeres como parte del proyecto. Esta inclusión nos permitía repensar y resituar la perspectiva del concepto de igualdad.

Simone de Beauvoir, reivindicaba que el trabajo era lo único que podía garantizar la libertad completa a una mujer. Se refería a una independencia económica. Por su parte, Amaia Pérez Orozco nos habla hoy de una subversión feminista de la economía, cuestionando la relación salarial misma y la estructura capitalista en su conjunto. En nuestro proyecto se trata de preguntarnos no sólo cómo lograr trabajo igualitario para todos y todas, sino para qué trabajamos. La economía feminista, enfocada en temas de cuidados y trabajo no remunerado, supone un reto que busca producir una inclusión de género. De ahí que esta economía defienda que los indicadores económicos, como el Producto Interior Bruto (PIB) no tengan en cuenta solo el trabajo mercantil, sino también el trabajo doméstico, fundamental para sostener la vida de las personas, aunque no posea una contrapartida económica. Cuestionar la división sexual del trabajo ha sido necesaria para hablar de igualdad porque esta división sexual en el trabajo y también en la educación, perpetúa los roles, estereotipos y desigualdades de genero. 
El proceso del proyecto, desglosado en dos actos fundamentales, enuncia dos acciones en relación al ser humano: la persona individual que es el yo; y la persona en comunidad que es la sociedad, y la sociedad en la globalización representada en las redes sociales. El cambio que busca el proyecto se inicia desde la autobiografía, en el propio currículum vitae, que representa el "quién soy yo y quién quiero ser".

Nuestra investigación se ha centrado en la violencia de género estructural ejercida desde la sociedad capitalista patriarcal hacia las mujeres y, en concreto, en los mecanismos por los que se perpetúan las discriminaciones hacia niñas y mujeres en el siglo XXI en el ámbito laboral. El proyecto constata cómo la división sexual del trabajo y la educación están ligadas a un sistema de creencias en el que persisten los roles y estereotipos del binarismo de género transmitidos a través de la cultura, la familia, las religiones, los estados, los medios de comunicación y actualmente las redes sociales.

Como conclusión final, el proyecto plantea, desde el feminismo activista, que toda nuestra percepción del mundo puede ser nombrada desde una perspectiva de género, lo que supone, en sí mismo, una propuesta formal de cambio radical en nuestro sistema educativo y en el ámbito universitario en especial. Al igual que la economía feminista, el proyecto busca producir una inclusión de género en todos los ámbitos de la práctica y del conocimiento.

*Mi especial agradecimiento a Carles Xavier López Benedí Director del Colegio Mayor Rector Peset, a M ${ }^{\text {a }}$ Trini Quixal Alejos Tècnica Cultural, y a todo el equipo. A Alba Braza, Michael Urrea Montoya y a todas las personas que han participado en la Campaña con la implicación de 10 países, a través de 18 personas entrañables: Xema, Irene, Josseline, Yulianne, Teslem, Lizeth, Evelyn, Álvaro, Rita, Máfer, Paola, Nicole, Jonathan, Fatine, Laura, Elimane, Alex y Marta.

\section{Referencias}

Сово, R. (2005). Globalización y nuevas servidumbres de las mujeres, <http://www.mujeresenred.net/spip.php?article385 > [Consulta: 14 de agosto de 2019]

HiLl, C., CoRbett, C. (2012). Graduating to a pay gap: The earnings of women and men one year after college graduation. Washington, DC: AAUW.

Monleón. M. (2017). Women in Work. Mujer, arte y trabajo en la globalización. Valencia: Universidad Politécnica de Valencia.

Monleón. M. (2015). “Arte participativo contra la violencia de género y la desigualdad entre los sexos.” en AAVV, Artistas, violencias, afectos, diálogos, creaciones. Zaragoza: Universidad de Zaragoza. Gobierno de Aragón. Pp: 9-17.

Monleón. M. (2015). "Feminicidio” en AAVV, Disculpen las molestias, el machismo mata. Valencia: UPV. Pp. 17-18.

MonleÓn. M. (2014). "Towards A Socio-Political Ethics Of Art And Technology In The Era Of Globalization. Fighting Gender Violence In The Public Sphere" en AAVV, Critical Cartography of Art and Visuality in the Global Age. Newcastle upon Tyne: Cambridge Scholars Publishing. Pp. 215 - 242.

PARello Rubio, S. (2004). Mujer, inmigrante y trabajadora: la triple discriminación. Madrid: Anthropos.

PÉREz OROZCO, A. (2014). Subversión feminista de la economía. Aportes para un debate sobre el conflicto capital-vida. Madrid: Traficantes de Sueños. 